\title{
Non-renormalizability of the classical statistical approximation
}

\author{
Thomas Epelbaum, François Gelis, Bin Wu
}

October 30, 2018

Institut de Physique Théorique (URA 2306 du CNRS)

CEA/DSM/Saclay, 91191 Gif-sur-Yvette Cedex, France

\begin{abstract}
In this paper, we discuss questions related to the renormalizability of the classical statistical approximation, an approximation scheme that has been used recently in several studies of out-of-equilibrium problems in Quantum Field Theory. Although the ultraviolet power counting in this approximation scheme is identical to that of the unapproximated quantum field theory, this approximation is not renormalizable. The leading cause of this non-renormalizability is the breakdown of Weinberg's theorem in this approximation. We also discuss some practical implications of this negative result for simulations that employ this approximation scheme, and we speculate about a possible modification of the classical statistical approximation in order to systematically subtract the leading residual divergences.
\end{abstract}

\section{Introduction}

In recent years, there has been a lot of interest in the study of out-of-equilibrium systems in quantum field theory, in view of applications to high energy heavy ion collisions, cosmology, or cold atom physics $[1,2,3,4,5,6,7,8,9,10,11,12,13$, $14,15,16,17,18,19,20,21,22,23,24,25,26,27,28,29,30,31,32,33]$. Generically, the question one would like to address is that of a system prepared in some non-equilibrium initial state, and let to evolve under the sole self-interactions of its constituents. The physically relevant quantum field theories cannot be solved exactly, and therefore some approximation scheme is mandatory in order to make progress. Moreover, the standard perturbative expansion in powers of the interaction strength is in general ill-suited to these out-of-equilibrium problems. Indeed, the coefficients in the perturbative expansion are time dependent and generically growing with time, thereby voiding the validity of the expansion after some finite time.

This "secularity" problem is resolved by the resummation of an infinite set of perturbative contributions, which can be achieved via several schemes. The 
simplest of these schemes is kinetic theory. However, in order to obtain a Boltzmann equation from the underlying quantum field theory, several important assumptions are necessary [34]: (i) a relatively smooth system, so that a gradient expansion can be performed, and (ii) the existence of well-defined quasiparticles. These limitations, especially the latter, make kinetic theory difficult to justify for describing the early stages of heavy ion collisions.

Closer to the underlying quantum field theory, two resummation schemes have been widely considered in many works. One of them is the 2-particle irreducible (2PI) approximation [35, 36, 37, 38, 39, 40, 41, 42, 43, 44, 28, 45, 46]. This scheme consists in solving the Dyson-Schwinger equations for the 2-point functions (and possibly for the expectation value of the field, if it differs from zero). The self-energy diagrams that are resummed on the propagator are obtained self-consistently from the sum of $2 \mathrm{PI}$ skeleton vacuum diagrams (often denoted $\Gamma_{2}[G]$ in the literature). The only approximation arises from the practical necessity of truncating the functional $\Gamma_{2}[G]$ in order to have manageable expressions. In applications, the $2 \mathrm{PI}$ scheme suffers from two limitations. One of them is purely computational: the convolution of the self-energy with the propagator takes the form of a memory integral, that in principle requires that one stores the entire history of the evolution of the system, from the initial time to the current time. The needed storage therefore grows quadratically with time ${ }^{1}$. The second difficulty appears in systems that are the siege of large fields, or large occupation numbers. For instance, in QCD, "large" would mean of order $g^{-1}$ for the fields, and of order $g^{-2}$ for the gluon occupation number. In this regime, the functional $\Gamma_{2}[G]$ contains terms that have the same order of magnitude at every order in the loop expansion, and therefore one cannot justify to truncate it at a finite loop order ${ }^{2}$. It turns out that these problems of strong fields occur in real world problems, e.g. in the early stages of heavy ion collisions [47, 48, 49, 50, 51].

There is an alternative resummation scheme, that includes all the leading contributions in the large field regime and is similarly free of secular terms, called the Classical Statistical Approximation (CSA) [52, 53, 54, 55, 56, 57]. It owes its name to the way it is implemented in practice, as an average over classical solutions of the field equations of motion, with a Gaussian statistical ensemble of initial conditions. The ability of this method to remain valid in the large field regime comes with a tradeoff : the CSA can be tuned to be exact at the 1-loop level, but starting at the 2-loop order and beyond, it includes only a subset of all the possible contributions. The CSA can be derived via several methods : from the path integral representation of observables [57], as an approximation at the level of the diagrammatic rules in the retarded-advanced formalism, or as an exponentiation of the 1-loop result [55].

\footnotetext{
${ }^{1}$ This can be alleviated somewhat by an extra approximation, in which one stores only the "recent" history of the system, in a sliding time window that moves with the current time.

${ }^{2}$ Such a truncation becomes legitimate, even in the large field regime, if there is an additional expansion parameter that one can use to control the loop expansion in $\Gamma_{2}[G]$. In some theories with a large number $N$ of constituents (e.g. an $O(N)$ scalar theory in the limit $N \rightarrow \infty$ ), one can compute exactly the leading term of $\Gamma_{2}[G]$ in the $1 / N$ expansion [40].
} 
The diagrammatic rules that define the classical statistical approximation allow graphs that have arbitrarily many loops. As in any field theory, the loops that arise in this expansion involve an integral over a 4-momentum, and this integral can be ultraviolet divergent. In the underlying -non approximated-field theory, we know how to deal with these infinities by redefining a finite number of parameters of the Lagrangian (namely the coupling constant, the mass and the field normalization). In general, this is done by first introducing an ultraviolet regulator, for instance a momentum cutoff $\Lambda_{\mathrm{UV}}$ on the loop momenta, and by letting the bare parameters of the Lagrangian depend on $\Lambda_{\mathrm{UV}}$ in such a way that physical quantities are independent of $\Lambda_{\mathrm{UV}}$ (and of course are finite in the limit $\left.\Lambda_{\mathrm{UV}} \rightarrow \infty\right)$. That this redefinition is possible is what characterizes a renormalizable field theory.

In contrast, non-renormalizable theories are theories in which one needs to introduce new operators that did not exist in the Lagrangian one started from, in order to subtract all the ultraviolet divergences that arise in the loop expansion. This procedure defines an "ultraviolet completion" of the original theory, which is well defined at arbitrary energy scales. The predictive power of the original theory is limited by the order at which it becomes necessary to introduce these new operators ${ }^{3}$.

It can also happen that, starting from a renormalizable field theory, certain approximations of this theory (for instance including certain loop corrections, but not all of them) are not renormalizable. This will be our main concern in this paper, in the context of the classical statistical approximation. A recent numerical study [5] showed a pronounced cutoff dependence for rather large couplings in a computation performed in this approximation scheme. This could either mean that the CSA is not renormalizable, or that the CSA is renormalizable but that renormalization was not performed properly in this computation. It is therefore of utmost importance to determine to which class -renormalizable or non-renormalizable- the CSA belongs, since this has far reaching practical implications on how it can be used in order to make predictive calculations, and how to interpret the existing computations.

Note that the question of the renormalizability of classical approximation schemes has already been discussed in quantum field theory at finite temperature $[58,59,60,61,62]$, following attempts to calculate non-perturbatively the sphaleron transition rate $[63,64,65,66,67,68,69,70]$. In this context, one is calculating the leading high temperature contribution, and in the classical approximation the Bose-Einstein distribution gets replaced by $T / \omega_{\boldsymbol{k}}$. This approximation leads to ultraviolet divergences in thermal contributions, that would otherwise be finite thanks to the exponential tail of the Bose-Einstein distribution. However, it has been shown that only a finite number of graphs have such divergences, and that they can all be removed by appropriate counterterms. The problem we will consider in this paper is different since we are interested in the classical approximation of a zero-temperature quantum field

\footnotetext{
${ }^{3}$ The predictive power of its ultraviolet completion may be quite limited as well, depending on how many new operators need to be introduced at each order (especially if this number grows very quickly or even worse becomes infinite).
} 
theory, where the factors $T / \omega_{\boldsymbol{k}}$ are replaced by $1 / 2$. This changes drastically the ultraviolet behavior.

In the section 2, we expose the scalar toy model we are going to use throughout the paper as a support of this discussion, we also remind the reader of the closed time path formalism and of the retarded-advanced formalism (obtained from the latter via a simple field redefinition), and we present the classical statistical approximation in two different ways (one that highlights its diagrammatic rules, and one that is more closely related to the way it is implemented in numerical simulations). Then, we analyze in the section 3 the ultraviolet power counting in the CSA, and show that it is identical to that in the underlying field theory. In the section 4, we examine all the one-loop 2-point and 4-point functions in the CSA, and we show that one of them violates Weinberg's theorem. This leads to contributions that are non-renormalizable in the CSA. In the section 5, we discuss the implications of non-renormalizability of the CSA for the calculation of some observables. We also argue that it may be possible to systematically subtract the leading non-renormalizable terms by the addition of a complex noise term to the classical equations of motion. Finally, the section 6 is devoted to concluding remarks. Some technical derivations are relegated into two appendices.

\section{Preliminaries}

\subsection{Toy model}

In order to illustrate our point, let us consider a massless real scalar field $\phi$ in four space-time dimensions, with quartic self-coupling, and coupled to an external source $j(x)$,

$$
\mathcal{L} \equiv \frac{1}{2}\left(\partial_{\mu} \phi\right)\left(\partial^{\mu} \phi\right)-\frac{m^{2}}{2} \phi^{2}-\frac{g^{2}}{4 !} \phi^{4}+j \phi .
$$

In this model, $j(x)$ is a real valued function, given once for all as a part of the description of the model. Sufficient regularity and compactness of this function will be assumed as necessary.

We also assume that the state of the system at $x^{0}=-\infty$ is the vacuum state (by adiabatically turning off the couplings at asymptotic times, we can assume that this is the perturbative vacuum state $\left.\left|0_{\text {in }}\right\rangle\right)$. Because of the coupling to the external source $j(x)$, the system is driven away from the vacuum state, and observables measured at later times acquire non-trivial values. Our goal is to compute the expectation value of such observables, expressed in terms of the field operator and its derivatives, in the course of the evolution of the system,

$$
\langle\mathcal{O}\rangle \equiv\left\langle 0_{\text {in }}|\mathcal{O}[\phi, \partial \phi]| 0_{\text {in }}\right\rangle .
$$

For simplicity, one may assume that the observable is a local (i.e. depends on the field operator at a single space-time point) or multi-local operator (i.e. depends on the field operator at a finite set of space-time points). 


\subsection{Closed time path formalism}

It is well known that the proper framework to compute expectation values such as the one defined in eq. (2) is the Schwinger-Keldysh (or "closed time path") formalism [71, 72]. In this formalism, there are two copies $\phi_{+}$and $\phi_{-}$of the field (corresponding respectively to fields in amplitudes and fields in complex conjugated amplitudes), and four bare propagators depending on which type of fields they connect. The expectation value of eq. (2) can be expanded diagrammatically (each loop brings an extra power of the coupling $g^{2}$ ) by a set of rules that generalize the traditional Feynman rules in a simple manner :

i. Each vertex of a graph can be of type + or - , and for a given graph topology one must sum over all the possible assignments of the types of these vertices. The rule for the + vertex $\left(-i g^{2}\right)$ and for the - vertex $\left(+i g^{2}\right)$ differ only in their sign. The same rule applies to the external source $j$.

ii. A vertex of type $\epsilon$ and a vertex of type $\epsilon^{\prime}$ must be connected by a bare propagator $G_{\epsilon \epsilon^{\prime}}^{0}$. In momentum space, these bare propagators read :

$$
\begin{array}{rlrl}
G_{++}^{0}(p) & =\frac{i}{p^{2}-m^{2}+i \epsilon}, & & G_{--}^{0}(p)=\frac{-i}{p^{2}-m^{2}-i \epsilon} \\
G_{+-}^{0}(p)=2 \pi \theta\left(-p^{0}\right) \delta\left(p^{2}-m^{2}\right), & G_{-+}^{0}(p) & =2 \pi \theta\left(p^{0}\right) \delta\left(p^{2}-m^{2}\right)
\end{array}
$$

The four bare propagators of the Schwinger-Keldysh formalism are related by a simple algebraic identity,

$$
G_{++}^{0}+G_{--}^{0}=G_{+-}^{0}+G_{-+}^{0}
$$

that one can check immediately from eqs. (3). Note that, on a more fundamental level, this identity follows from the definition of the various $G_{\epsilon \epsilon^{\prime}}$ as vacuum expectation values of pairs of fields ordered in various ways. For this reason, it is true not only for the bare propagators, but for their corrections at any order in $g^{2}$.

\subsection{Retarded-advanced formalism}

The Schwinger-Keldysh formalism is not the only one that can be used to calculate eq. (2). One can arrange the four bare propagators $G_{\epsilon \epsilon^{\prime}}^{0}$ in a $2 \times 2$ matrix, and obtain equivalent diagrammatic rules by applying a "rotation" to this matrix $[73,74,75,61]$. Among this family of transformations, especially interesting are those that exploit the linear relationship (4) among the $G_{\epsilon \epsilon^{\prime}}^{0}$ in order to obtain a vanishing entry in the rotated matrix. The retarded-advanced formalism belongs to this class of transformations, and its propagators are defined by (let us denote $\alpha=1,2$ the two values taken by the new index) :

$$
\mathbb{G}_{\alpha \beta}^{0} \equiv \sum_{\epsilon, \epsilon^{\prime}= \pm} \Omega_{\alpha \epsilon^{\prime}} \Omega_{\beta \epsilon^{\prime}} G_{\epsilon \epsilon^{\prime}}^{0}
$$


with the transformation matrix defined as

$$
\Omega_{\alpha \epsilon} \equiv\left(\begin{array}{cc}
1 & -1 \\
1 / 2 & 1 / 2
\end{array}\right)
$$

The bare rotated propagators read

$$
\mathbb{G}_{\alpha \beta}^{0}=\left(\begin{array}{cc}
0 & G_{A}^{0} \\
G_{R}^{0} & G_{S}^{0}
\end{array}\right)
$$

where we have introduced

$$
G_{R}^{0}=G_{++}^{0}-G_{+-}^{0}, G_{A}^{0}=G_{++}^{0}-G_{-+}^{0}, G_{S}^{0}=\frac{1}{2}\left(G_{++}^{0}+G_{--}^{0}\right) .
$$

(The subscripts R, A and S stand respectively for retarded, advanced and symmetric.)

It is straightforward to verify that in the rotated formalism, the various vertices read :

$$
\Gamma_{\alpha \beta \gamma \delta} \equiv-i g^{2}\left[\Omega_{+\alpha}^{-1} \Omega_{+\beta}^{-1} \Omega_{+\gamma}^{-1} \Omega_{+\delta}^{-1}-\Omega_{-\alpha}^{-1} \Omega_{-\beta}^{-1} \Omega_{-\gamma}^{-1} \Omega_{-\delta}^{-1}\right],
$$

where

$$
\Omega_{\epsilon \alpha}^{-1}=\left(\begin{array}{cc}
1 / 2 & 1 \\
-1 / 2 & 1
\end{array}\right) \quad\left[\Omega_{\alpha \epsilon} \Omega_{\epsilon \beta}^{-1}=\delta_{\alpha \beta}\right] .
$$

More explicitly, we have :

$$
\begin{aligned}
& \Gamma_{1111}=\Gamma_{1122}=\Gamma_{2222}=0 \\
& \Gamma_{1222}=-i g^{2}, \quad \Gamma_{1112}=-i g^{2} / 4 .
\end{aligned}
$$

(The vertices not listed explicitly here are obtained by trivial permutations.) Concerning the insertions of the external source, the diagrammatic rules in the retarded-advanced formalism are :

$$
J_{1}=i j, \quad J_{2}=0 .
$$

\subsection{From the external source to an external classical field}

From the above rules, we see that an external source can only be attached to a propagator endpoint of type 1 , i.e. to the lowest time endpoint of a retarded or advanced propagator $\left(G_{12}^{0}=G_{A}^{0}, G_{21}^{0}=G_{R}^{0}\right)$, as in the formula

$$
\int \mathrm{d}^{4} y G_{21}^{0}(x, y) J_{1}(y) .
$$

(This expression corresponds to the first graph on the left of the figure 1.) It is easy to see that the external source can be summed to all orders, if one introduces the object $\varphi(x)$ defined diagrammatically in the figure 1. It is well 


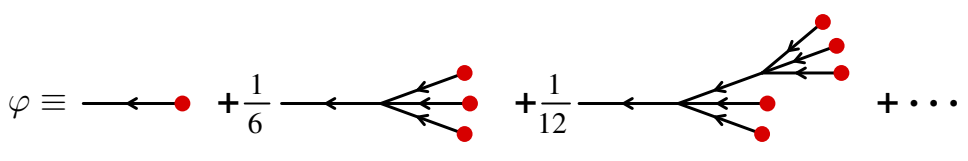

Figure 1: The first three terms of the diagrammatic expansion of the external field in terms of the external source, for a field theory with quartic coupling. The red dots represent the source insertions $J_{1}$, and the lines with an arrow are bare retarded propagators $G_{21}^{0}$. The quartic vertices are all of the type $\Gamma_{1222}$.

known that this series obeys the classical equation of motion,

$$
\left(\square+m^{2}\right) \varphi+\frac{g^{2}}{6} \varphi^{3}=j,
$$

and since all the propagators are all of type $G_{21}^{0}$, i.e. retarded, it obeys the following boundary condition :

$$
\lim _{x^{0} \rightarrow-\infty} \varphi(x)=0 .
$$

The source $j$ can be completely eliminated from the diagrammatic rules, by adding to the Lagrangian couplings between the field operator $\phi$ and the classical field $\varphi$,

$$
\Delta \mathcal{L} \equiv g^{2}\left[\frac{1}{2} \varphi^{2} \phi_{1} \phi_{2}+\frac{1}{2} \varphi \phi_{1} \phi_{2}^{2}+\frac{1}{4 !} \varphi \phi_{1}^{3}\right] .
$$

Note that, since in all the graphs in the figure 1 the root of the tree is terminated by an index 2 , the classical field $\varphi$ can only be attached to an index of type 2 in these vertices.

\subsection{Classical statistical approximation (CSA)}

\subsubsection{Definition from truncated retarded-advanced rules}

The classical statistical approximation consists in dropping all the graphs that contain the vertex $\Gamma_{2111}$, i.e. in assuming :

$$
\Gamma_{2111}=0 \quad \text { (and similarly for the permutations of 2111) . }
$$

In the rest of this paper, we will simply call CSA the field theory obtained by dropping all the vertices that have 3 indices of type 1 in the retarded-advanced formalism, while everything else remains unchanged. Therefore, in the retardedadvanced formalism, the CSA is defined by the following diagrammatic rules :

\section{i. bare propagators :}

$$
\begin{aligned}
& G_{21}^{0}(p)=\frac{i}{\left(p^{0}+i \epsilon\right)^{2}-p^{2}-m^{2}}, \quad G_{12}^{0}(p)=\frac{i}{\left(p^{0}-i \epsilon\right)^{2}-p^{2}-m^{2}}, \\
& G_{22}^{0}(p)=\pi \delta\left(p^{2}-m^{2}\right) .
\end{aligned}
$$




\section{ii. vertices :}

$$
\Gamma_{1222}(\text { and permutations })=-i g^{2} \quad, \quad \text { all other combinations zero . }
$$

\section{iii. external sources :}

$$
J_{1}=i j, \quad J_{2}=0
$$

iii'. external field (see the section 2.4) :

$$
\Phi_{1}=0, \quad \Phi_{2}=\varphi
$$

Note that this "truncated" field theory is still quite non trivial, in the sense that the above diagrammatic rules allow graphs with arbitrarily many loops. The numerical simulations that implement the CSA provide the sum to all orders of the graphs that can be constructed with these rules (with an accuracy in principle only limited by the statistical errors in the Gaussian average over the initial field fluctuations, since this average is approximated by a Monte-Carlo sampling).

\subsubsection{Definition by exponentiation of the 1-loop result}

The previous definition of the CSA makes it very clear what graphs are included in this approximation and what graphs are not. However, it is a bit remote from the actual numerical implementation. Let us also present here an alternative -but strictly equivalent- way of introducing the classical statistical approximation, that directly provides a formulation that can be implemented numerically.

Firstly, observables at leading order are expressible in terms of the retarded classical field $\varphi$ introduced above,

$$
\left\langle 0_{\text {in }}|\mathcal{O}[\phi, \partial \phi]| 0_{\text {in }}\right\rangle_{\text {LO }}=\mathcal{O}[\varphi, \partial \varphi] .
$$

At next-to-leading order, it has been shown in $[55,76,77]$ that the observable can be expressed as follows,

$$
\begin{aligned}
& \left\langle 0_{\text {in }}|\mathcal{O}[\phi, \partial \phi]| 0_{\text {in }}\right\rangle_{\mathrm{NLO}}= \\
& \quad=\left[\frac{1}{2} \int \frac{\mathrm{d}^{3} \boldsymbol{k}}{(2 \pi)^{3} 2 E_{\boldsymbol{k}}} \int \mathrm{d}^{3} \boldsymbol{u} \mathrm{d}^{3} \boldsymbol{v}\left(\boldsymbol{\alpha}_{\boldsymbol{k}} \cdot \mathbb{T}_{\boldsymbol{u}}\right)\left(\boldsymbol{\alpha}_{\boldsymbol{k}}^{*} \cdot \mathbb{T}_{\boldsymbol{v}}\right)\right] \mathcal{O}[\varphi, \partial \varphi]
\end{aligned}
$$

In eq. (23), the operator $\mathbb{T}_{\boldsymbol{u}}$ is the generator of shifts of the initial condition for the classical field $\varphi$ on some constant time surface (the integration surface for the variables $\boldsymbol{u}$ and $\boldsymbol{v}$ ) located somewhere before the source $j$ is turned on ${ }^{4}$.

\footnotetext{
${ }^{4}$ This is why eq. (23) does not have a term linear in $\mathbb{T}_{\boldsymbol{u}}$, contrary to the slightly more general formulas derived in refs. [55, 76, 77].
} 
This means that if we denote $\varphi\left[\varphi_{\text {init }}\right]$ the classical field as a functional of its initial condition, then for any functional $F[\varphi]$ of $\varphi$, we have

$$
\left[\exp \int \mathrm{d}^{3} \boldsymbol{u}\left(a \cdot \mathbb{T}_{\boldsymbol{u}}\right)\right] F\left[\varphi\left[\varphi_{\text {init }}\right]\right]=F\left[\varphi\left[\varphi_{\text {init }}+a\right]\right] .
$$

(This equation can be taken as the definition of $\mathbb{T}_{\boldsymbol{u}}$.) In eq. (23), the fields $\boldsymbol{\alpha}_{\boldsymbol{k}}$ are free plane waves of momentum $k^{\mu}$ :

$$
\boldsymbol{\alpha}_{\boldsymbol{k}}(u) \equiv e^{i k \cdot u}, \quad\left(\square+m^{2}\right) \boldsymbol{\alpha}_{k}(u)=0 .
$$

Note that in eq. (23), the integration variable $\boldsymbol{k}$ is a loop momentum. In general, the integral over $\boldsymbol{k}$ therefore diverges in the ultraviolet, and must be regularized by a cutoff. After the Lagrangian parameters have been renormalized at 1-loop, this cutoff can be safely sent to infinity.

In this framework, the classical statistical method is defined as the result of the exponentiation of the operator that appears in the right hand side of eq. (23),

$$
\begin{aligned}
& \left\langle 0_{\text {in }}|\mathcal{O}[\phi, \partial \phi]| 0_{\text {in }}\right\rangle_{\mathrm{CSA}}= \\
& \quad=\exp \left[\frac{1}{2} \int \frac{\mathrm{d}^{3} \boldsymbol{k}}{(2 \pi)^{3} 2 E_{\boldsymbol{k}}} \int \mathrm{d}^{3} \boldsymbol{u} \mathrm{d}^{3} \boldsymbol{v}\left(\boldsymbol{\alpha}_{\boldsymbol{k}} \cdot \mathbb{T}_{\boldsymbol{u}}\right)\left(\boldsymbol{\alpha}_{\boldsymbol{k}}^{*} \cdot \mathbb{T}_{\boldsymbol{v}}\right)\right] \mathcal{O}[\varphi, \partial \varphi]
\end{aligned}
$$

Note that by construction, the CSA is identical to the underlying theory at LO and NLO, and starts differing from it at NNLO and beyond (some higher loop graphs are included but not all of them). The relation between this formula and the way the classical statistical method is implemented lies in the fact that the exponential operator is equivalent to a Gaussian average over a Gaussian distribution of initial conditions for the classical field $\varphi$,

$$
\begin{gathered}
\exp \left[\frac{1}{2} \int \frac{\mathrm{d}^{3} \boldsymbol{k}}{(2 \pi)^{3} 2 E_{\boldsymbol{k}}} \int \mathrm{d}^{3} \boldsymbol{u} \mathrm{d}^{3} \boldsymbol{v}\left(\boldsymbol{\alpha}_{\boldsymbol{k}} \cdot \mathbb{T}_{\boldsymbol{u}}\right)\left(\boldsymbol{\alpha}_{\boldsymbol{k}}^{*} \cdot \mathbb{T}_{\boldsymbol{v}}\right)\right] F\left[\varphi\left[\varphi_{\text {init }}\right]\right] \\
=\int[\operatorname{Da}(\boldsymbol{u}) \operatorname{D} \dot{a}(\boldsymbol{u})] G[a, \dot{a}] F\left[\varphi\left[\varphi_{\text {init }}+a\right]\right]
\end{gathered}
$$

where $G[a, \dot{a}]$ is a Gaussian distribution, whose elements can be generated as

$$
a(u)=\int \frac{\mathrm{d}^{3} \boldsymbol{k}}{(2 \pi)^{3} 2 E_{\boldsymbol{k}}}\left[c_{\boldsymbol{k}} \boldsymbol{\alpha}_{\boldsymbol{k}}(u)+c_{\boldsymbol{k}}^{*} \boldsymbol{\alpha}_{\boldsymbol{k}}^{*}(u)\right],
$$

with $c_{\boldsymbol{k}}$ complex Gaussian random numbers defined by

$$
\left\langle c_{\boldsymbol{k}}\right\rangle=0 \quad, \quad\left\langle c_{\boldsymbol{k}} c_{\boldsymbol{l}}\right\rangle=0 \quad, \quad\left\langle c_{\boldsymbol{k}} c_{\boldsymbol{l}}^{*}\right\rangle=(2 \pi)^{3} E_{\boldsymbol{k}} \delta(\boldsymbol{k}-\boldsymbol{l}) .
$$

We will not show here the equivalence of the two ways of defining the classical statistical approximation that we have exposed in this section. The main reason for recalling the second definition of the CSA was to emphasize the meaning of the variable $\boldsymbol{k}$ in eqs. (23), (26) and (28), as a loop momentum. Therefore, 
an upper limit introduced in the $\boldsymbol{k}$-integration in any of these formulas will effectively play the role of an ultraviolet cutoff that regularizes loop integrals.

To make the connection with the diagrammatic rules of the classical statistical approximation introduced in the previous subsection, the cutoff on the momentum of the initial fluctuations is an upper limit for the momentum flowing through the $G_{22}^{0}$ propagators. In contrast, the largest momentum that can flow through the $G_{21}^{0}$ and $G_{12}^{0}$ propagators is only controlled by the discretization of space, i.e. by the inverse lattice cutoff. In some implementations, these two cutoffs are identical, but other implementations have chosen to have distinct cutoffs for these two purposes ${ }^{5}$ :

- In Refs. $[2,3,4]$ an explicit cutoff $\Lambda_{\mathrm{UV}}$, distinct from the lattice cutoff, is introduced in order to limit the largest $\boldsymbol{k}$ of the initial fluctuations. In this setup, $\Lambda_{\mathrm{UV}}$ is smaller than the lattice momentum cutoff, and the lattice spacing no longer controls the ultraviolet limit of the computation.

- In Refs. $[13,14,15,17,5]$ fluctuation modes are included up to the lattice momentum cutoff, i.e. $\Lambda_{\mathrm{UV}}$ is inversely proportional to the lattice spacing.

A common caveat of most of these computations is that none has studied the behavior of the results in the limit $\Lambda_{\mathrm{UV}} \rightarrow \infty$, at the exception of ref. [5] where a strong dependence on the ultraviolet cutoff was found.

\section{Ultraviolet power counting}

After having defined the classical statistical approximation, we can first calculate the superficial degree of ultraviolet divergence for arbitrary graphs in the CSA, in order to see what kind of divergences one may expect. This is best done by using the definition introduced in the section 2.5.1, that defines the CSA by its diagrammatic rules.

Let us consider a generic connected graph $\mathcal{G}$ built with these diagrammatic rules, made of :

- $E$ external legs

- I internal lines

- $L$ independent loops

- $V$ vertices of type $\phi^{4}$

- $V_{2}$ vertices of type $\phi^{2} \varphi^{2}$

- $V_{1}$ vertices of type $\phi^{3} \varphi$

${ }^{5}$ The downside of having two separate cutoffs is that this form of regularization violates [78] the Kubo-Martin-Schwinger identities [79, 80], which leads to a non-zero scattering rate even in the vacuum. 
Note that for the internal lines, the superficial degree of divergence does not distinguish ${ }^{6}$ between the propagators $G_{12}^{0}, G_{21}^{0}$ and $G_{22}^{0}$, because they all have a mass dimension -2 . These numbers are related by the following relations :

$$
\begin{aligned}
E+2 I & =4 V+3 V_{1}+2 V_{2}, \\
L & =I-\left(V+V_{1}+V_{2}\right)+1 .
\end{aligned}
$$

The first of these identities states that the number of propagator endpoints must be equal to the number of slots where they can be attached to vertices. The second identity counts the number of independent momenta that can circulate in the loops of the graph.

In terms of these quantities, the superficial degree of divergence of the graph $\mathcal{G}$ is given by

$$
\begin{aligned}
\omega(\mathcal{G}) & =4 L-2 I \\
& =4-E-\left(V_{1}+2 V_{2}\right) \\
& =4-E-N_{\varphi},
\end{aligned}
$$

where $N_{\varphi} \equiv V_{1}+2 V_{2}$ is the number of powers of the external classical field $\varphi$ inserted into the graph $\mathcal{G}$.

Note that $4-E$ is the superficial degree of divergence of a graph with $E$ external points in a 4-dimensional scalar $\phi^{4}$ theory, in the absence of an external field/source. Therefore, the external field can only decrease the superficial degree of divergence (since $N_{\varphi} \geq 0$ ), which was expected since the couplings to the external field (see eq. (16)) have a positive mass dimension, i.e. they are super-renormalizable interactions.

The crucial point about this formula is that the superficial degree of divergence does not depend on the fact that we have excluded the vertices of type 2111 in the classical statistical approximation. In other words, the ultraviolet power counting is exactly the same in the full theory and in the CSA. Eq. (32) suggests that the only ultraviolet divergent quantities are those for which $E \leq 4$, exactly as in the unapproximated theory.

As we shall see in the next section, there is nevertheless an issue that hinders the renormalizability of the classical statistical approximation. Discarding the $\Gamma_{1112}$ alters in subtle ways the analytic structure of Green's functions, which leads to a violation of Weinberg's theorem ${ }^{7}$. As a consequence, ultraviolet divergences in the CSA can be stronger that one would expect on the basis of the power counting alone.

\footnotetext{
${ }^{6}$ As we shall see later, due to the peculiar analytic structure of the integrands of graphs in the CSA, this power counting is too naive to accurately reflect the actual ultraviolet divergences.

${ }^{7}$ In addition to power counting arguments, renormalizability requires some handle on the recursive structure of the ultraviolet divergences. This may come in the form of Dyson's convergence theorem [81], whose proof was completed by Weinberg [82] and somewhat simplified by Hahn and Zimmermann [83, 84] (see also Refs. [85, 86]). This result states that if all the divergences in the subgraphs of a given graph $\mathcal{G}$ have been subtracted, then the remaining divergence is a polynomial of degree $\omega(\mathcal{G})$ in the external momenta.
} 


\section{Ultraviolet divergences in the CSA}

\subsection{Introduction}

The un-truncated $\phi^{4}$ theory that we started from is well known to be renormalizable ${ }^{8}$. This means that all its ultraviolet divergences can be disposed of by redefining the coefficients in front of the operators that appear in the bare Lagrangian.

In the retarded-advanced basis, the Lagrangian of the CSA differs from the Lagrangian of the unapproximated theory in the fact that the vertex $\Gamma_{1112}$ is missing. All the other terms of the Lagrangian are unchanged, in particular the operators that are quadratic in the fields. In this section we systematically examine 2- and 4-point functions at one loop, in order to see whether their ultraviolet behavior is compatible with renormalizability or not.

\subsection{Self-energies at one loop}

Let us start with the simplest possible loop correction: the one-loop self-energy, made of a tadpole graph. Depending on the indices 1 and 2 assigned to the two external legs, these self-energies are given in eq. (33).

$$
-i\left[\Sigma_{11}\right]_{\mathrm{CSA}}^{1 \text { loop }}=0, \quad-i\left[\Sigma_{22}\right]_{\mathrm{CSA}}^{1 \text { loop }}=\frac{2}{\mathbf{2}}=0
$$

$\Sigma_{11}$ is zero at one loop in the CSA, because it requires a vertex 1112 that has been discarded. $\Sigma_{22}$ is also zero, because it contains a closed loop made of a retarded propagator. The only non-zero self-energy at 1-loop is $\Sigma_{12}$, that displays the usual quadratic divergence. This can be removed by a mass counterterm in the Lagrangian,

$$
\delta m^{2}=-\frac{g^{2} \Lambda_{\mathrm{UV}}^{2}}{16 \pi^{2}},
$$

since the mass term in the Lagrangian is precisely a $\phi_{1} \phi_{2}$ operator.

\subsection{Four point functions at one loop}

\subsubsection{Vanishing functions : $\Gamma_{1112}, \Gamma_{1111}$ and $\Gamma_{2222}$}

The 4-point function with indices 1112 is a prime suspect for Green's functions that may cause problems with the renormalizability of the CSA. Indeed, the

${ }^{8}$ The fact that we are dealing here with a field theory coupled to an external source does not spoil this property, for sufficiently smooth external sources. See Ref. [87], chapter 11. 
CSA consists in discarding the operator corresponding to this vertex from the Lagrangian. Therefore, if an intrinsic ${ }^{9}$ ultraviolet divergent contribution to this Green's function can be generated in the classical statistical approximation, then the CSA is not renormalizable.

Let us first consider this 4-point function at 1-loop. At this order, the only possible contribution (up to trivial permutations of the external legs) to the $\Gamma_{1112}$ function is

$$
-i \Gamma_{1112}^{1 \text { loop }}=\sum_{12}^{11}
$$

where the indices 1 and 2 indicate the various vertex assignments. (The $-i$ prefactor is a convention, so that the function $\Gamma$ can be viewed directly as a correction to the coupling constant $g^{2}$.) Because it must contain a vertex of type 1112 , this function is zero in the classical statistical approximation ${ }^{10}$,

$$
-i\left[\Gamma_{1112}\right]_{\mathrm{CSA}}^{1 \text { loop }}=0 \text {. }
$$

Therefore, this 4-point function does not cause any renormalization problem in the CSA at 1-loop. Similarly, the function $\Gamma_{1111}$ at one loop also requires the vertex 1112 , and is therefore zero in the classical statistical approximation ${ }^{11}$,

$$
-i\left[\Gamma_{1111}\right]_{\mathrm{CSA}}^{1 \text { loop }}=0 \text {. }
$$

For the function $\Gamma_{2222}$ at one loop, the only possibility is the following,

$$
-i\left[\Gamma_{2222}\right]_{\mathrm{CSA}}^{1 \text { loop }}=\overbrace{2 \mathrm{~T}}^{22}=0,
$$

where we have represented with arrows the 12 propagators, since they are retarded propagators. This graphs is zero because it is made of a sequence of retarded propagators forming a closed loop.

\subsubsection{Logarithmic divergence in $\Gamma_{1222}$}

At one loop, the function $\Gamma_{1222}$ is given by the graph of eq. (39) (and several other permutations of the indices).

$$
-i\left[\Gamma_{1222}\right]_{\mathrm{CSA}}^{1 \text { loop }}=>_{12}^{22} \sim g^{4} \log \left(\Lambda_{\mathrm{UV}}\right) .
$$

It is a straightforward calculation to check that this graph has a logarithmic ultraviolet divergence, that can be removed by the standard 1-loop renormalization of the coupling constant (this is possible, since the interaction term $\phi_{1} \phi_{2}^{3}$

\footnotetext{
${ }^{9}$ Here, we are talking about the overall divergence of the function, not the divergences associated to its various subgraphs, that may be subtracted by having renormalized the other operators of the Lagrangian.

${ }^{10}$ For the calculation of the full $\Gamma_{1112}$ at one loop, beyond the classical statistical approximation, see the appendix A.

${ }^{11} \mathrm{At}$ one loop, the functions $\Gamma_{1111}$ and $\Gamma_{2222}$ are also zero in the full theory.
} 
has been kept in the Lagrangian when doing the classical statistical approximation). The calculation of this 4-point function is detailed in the appendix A.

\subsubsection{Violation of Weinberg's theorem in $\Gamma_{1122}$}

Another interesting object to study is the 4-point function with indices 1122 . There is no such bare vertex in the Lagrangian (both for the unapproximated theory and for the classical statistical approximation). Since the full theory is renormalizable, this function should not have ultraviolet divergences at 1-loop, since such divergences would not be renormalizable. However, since the CSA discards certain terms, it not obvious a priori that this conclusion still holds. For the sake of definiteness, let us denote $p_{1}, \cdots, p_{4}$ the external momenta of this function (defined to be incoming into the graph, therefore $p_{1}+p_{2}+p_{3}+p_{4}=0$ ), and let us assume that the two indices 1 are attached to the legs $p_{1}, p_{2}$ and the two indices 2 are attached to the legs $p_{3}, p_{4}$. At one loop, this 4-point function (in the full field theory) receives the following contributions :

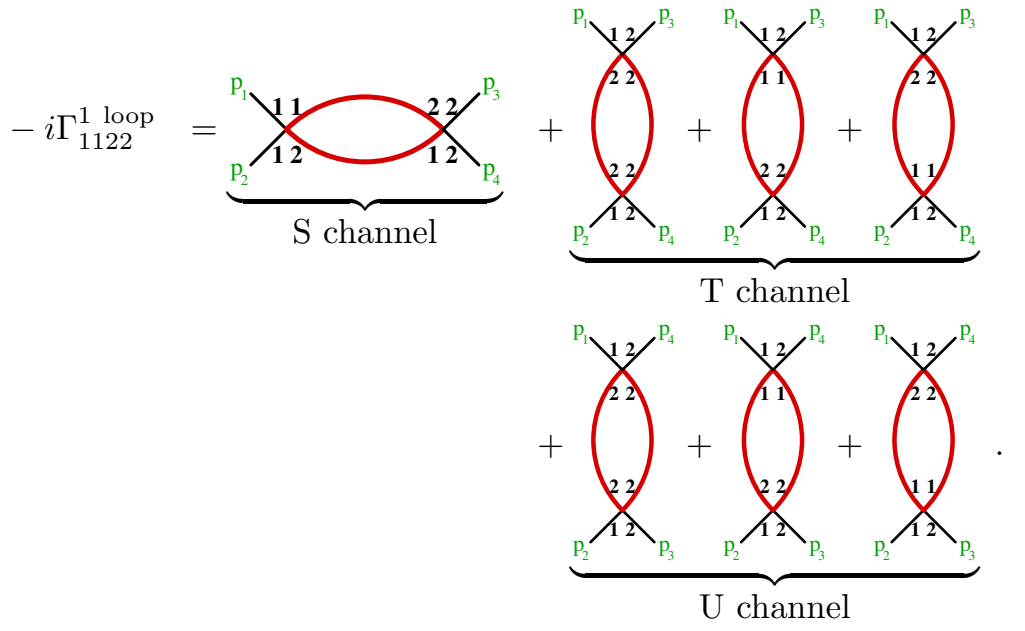

Since the full theory is renormalizable, the sum of all these graphs should be ultraviolet finite, because there is no 1122 4-field operator in the bare Lagrangian.

It is however not obvious that the subset of these graphs that exist in the classical statistical approximation is itself ultraviolet finite. Among the T-channel and U-channel graphs, only the first of the three graphs exist in the CSA, since all the other graphs contain the 1112 bare vertex,

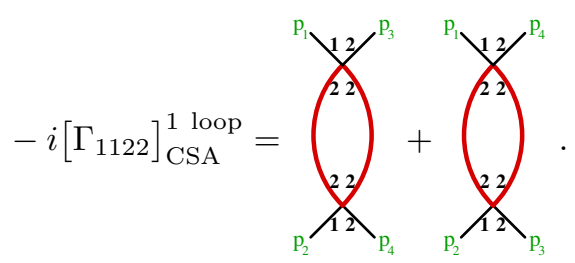


Some details of the calculation of these graphs are provided in the appendix B. One obtains

$-i\left[\Gamma_{1122}\right]_{\mathrm{CSA}}^{1 \text { loop }}=-\frac{g^{4}}{64 \pi}\left[\operatorname{sign}(T)+\operatorname{sign}(U)+2 \Lambda_{\mathrm{UV}}\left(\frac{\theta(-T)}{\left|\boldsymbol{p}_{1}+\boldsymbol{p}_{3}\right|}+\frac{\theta(-U)}{\left|\boldsymbol{p}_{1}+\boldsymbol{p}_{4}\right|}\right)\right]$,

where we denote

$$
T \equiv\left(p_{1}+p_{3}\right)^{2} \quad, \quad U \equiv\left(p_{1}+p_{4}\right)^{2},
$$

and where $\Lambda_{\mathrm{UV}}$ is an ultraviolet cutoff introduced to regularize the integral over the 3-momentum running in the loop. As one sees, these graphs have a linear ultraviolet divergence, despite having a superficial degree of divergence equal to zero. This property violates Weinberg's theorem since, if it were applicable here, it would imply at most a logarithmic divergence with a coefficient independent of the external momenta. One can attribute this violation to the analytic structure of the integrand ${ }^{12}$ : unlike in ordinary Feynman perturbation theory, we cannot perform a Wick rotation to convert the integral to an integral over an Euclidean momentum, which is an important step in the proof of Weinberg's theorem.

Since it occurs in the operator $\phi_{1}^{2} \phi_{2}^{2}$, that does not appear in the CSA Lagrangian, this linear divergence provides incontrovertible proof of the fact that the classical statistical approximation is not renormalizable. Moreover, this conclusion is independent of the value of the coupling constant. The only thing one gains at smaller coupling is that the irreducible cutoff dependence caused by these terms is weaker.

It should also be noted that this linear divergence is a purely imaginary contribution to the function $\Gamma_{1122}$ (this can be understood from the structure of the integrand, that was made of two delta functions, which is reminiscent of the calculation of the imaginary part of a Green's function via Cutkosky's cutting rules $[88,89])$.

In the appendix B, we also calculate the graphs of eq. (40) that do not contribute to the CSA, and we find

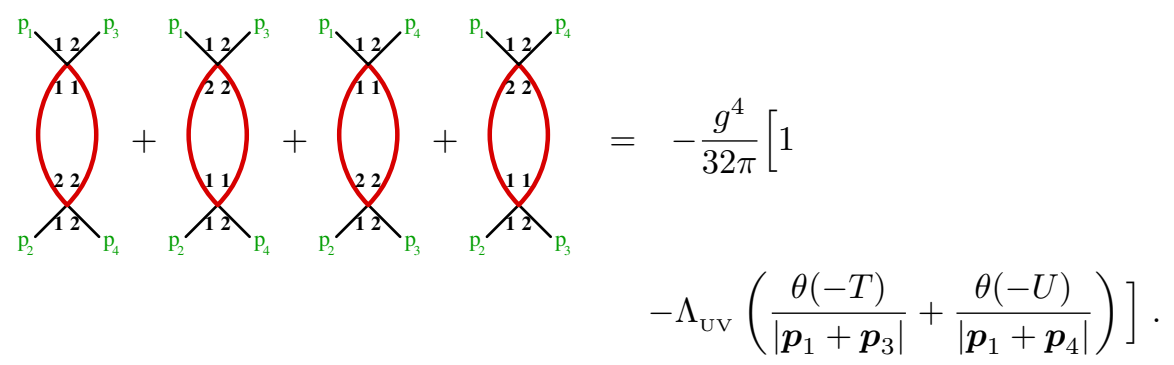

\footnotetext{
${ }^{12}$ For the graphs in eq. (41), the integrand is of the form $\delta\left(K^{2}\right) \delta\left((P+K)^{2}\right)$ where $P \equiv$ $p_{1}+p_{3}$ or $P \equiv p_{1}+p_{4}$. Using the first delta function, the argument of the second one is $(P+K)^{2}=2 P \cdot K+P^{2}$, which is only of degree 1 in the loop momentum. Therefore, the second propagator contributes only -1 to the actual degree of divergence of the graph, contrary to the -2 assumed based on dimensionality when computing the superficial degree of divergence. This discrepancy is also related to the impossibility to perform a Wick's rotation when the integrand is expressed in terms of delta functions or retarded/advanced propagators.
} 
(Note that the S-channel graph is in fact zero, because it is made of a sequence of retarded propagators in a closed loop.) By adding eqs. (42) and (44), we obtain the 1-loop result in the unapproximated theory,

$$
-i \Gamma_{1122}^{1 \text { loop }}=-\frac{g^{4}}{32 \pi}[\theta(T)+\theta(U)],
$$

which is ultraviolet finite, in agreement with the renormalizability of the full theory.

\subsection{Two point functions at two loops}

Let us also mention two problematic 2-point functions at two loops. We just quote the results here (the derivation will be given in [78]), for an on-shell momentum $P\left(P^{2}=0, p_{0}>0\right)$ :

$$
\begin{aligned}
-i\left[\Sigma_{11}(P)\right]_{\mathrm{CSA}}^{2 \text { loop }} & =\frac{g^{4}}{1024 \pi^{3}}\left(\Lambda_{\mathrm{UV}}^{2}-\frac{2}{3} p^{2}\right), \\
& =-\frac{1}{2} \mathbf{2} \\
\operatorname{Im}\left[\Sigma_{12}(P)\right]_{\mathrm{CSA}}^{2 \text { loop }} & =-\frac{g^{4}}{1024 \pi^{3}}\left(\Lambda_{\mathrm{UV}}^{2}-\frac{2}{3} p^{2}\right) .
\end{aligned}
$$

An ultraviolet divergence in $\Sigma_{11}$ is non-renormalizable, since there is no $\phi_{1}^{2}$ operator in the Lagrangian. Similarly, the divergence at 2-loops in $\operatorname{Im} \Sigma_{12}$ is also non-renormalizable, because it would require an imaginary counterterm, that would break the Hermiticity of the Lagrangian.

\section{Consequences on physical observables}

\subsection{Order of magnitude of the pathological terms}

So far, we have exhibited a 4-point function at 1-loop that has an ultraviolet divergence in the CSA but not if computed in full, and that cannot be renormalized in the CSA because it would require a counterterm for an operator that does not exist in the Lagrangian.

In practice, this 1-loop function enters as a subdiagram in the loop expansion of observable quantities, making them unrenormalizable. In order to assess the damage, it is important to know the lowest order at which this occurs. Let us consider in this discussion two quantities that have been commonly 
computed with the classical statistical method: the expectation value of the energy-momentum tensor, and the occupation number, which can be extracted from the $G_{22}$ propagator.

For the 22 component of the propagator, the first occurrence of the 1122 4-point function as a subgraph is in the following 1-loop contribution :

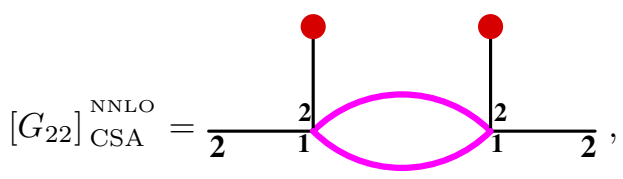

where the problematic subdiagram has been highlighted (the propagators in purple are of type $G_{22}$ ). The fact that this problematic subgraphs occurs only at 1-loop and beyond is related to the fact that classical statistical approximation is equivalent to the full theory up to (including) NLO. The first differences appear at NNLO, which means 1-loop for the $G_{22}$ function. In a situation where the typical physical scale is denoted $Q$, the subdiagram is of order $g^{4} \Lambda_{\mathrm{UV}} / Q$, and the external field attached to the graph is of order $\Phi_{2} \sim Q / g$ (we assume a system dominated by strong fields, as in applications to heavy ion collisions). This 1-loop contribution to $G_{22}$ is of order $g^{2} \Lambda_{\mathrm{UV}} Q$, to be compared to $Q^{2} / g^{2}$ at leading order. Therefore, the relative suppression of this non-renormalizable contribution is by a factor

$$
g^{4} \frac{\Lambda_{\mathrm{UV}}}{Q} .
$$

The same conclusion holds in the case of the energy-momentum tensor, for which the 1122 4-point function enters also at NNLO (in this case, this means two loops), in the following diagram :

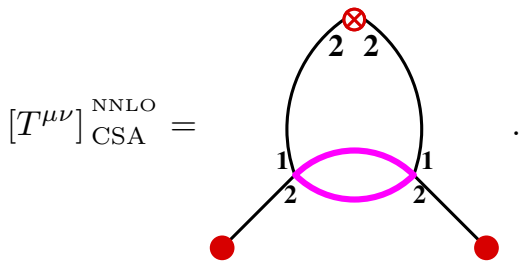

(The cross denotes the insertion of the $T^{\mu \nu}$ operator.) The order of magnitude of this graph is $g^{2} \Lambda_{\mathrm{UV}} Q^{3}$, while the leading order contribution to the energymomentum tensor is of order $Q^{4} / g^{2}$. Therefore, the relative suppression is the same as in eq. (49).

All these examples suggest that a minimum requirement is that the ultraviolet cutoff should satisfy

$$
\Lambda_{\mathrm{UV}} \ll \frac{Q}{g^{4}},
$$

for the above contributions to give only a small contamination to their respective observables in a classical statistical computation with cutoff $\Lambda_{\mathrm{UV}}$. However, one could be a bit more ambitious and request that this computation be also 
accurate at NLO. For this, we should set the cutoff so that the above diagrams are small corrections compared to the NLO contributions. This is achieved if the highlighted 4-point function in these graphs is small compared to the tree-level 4-point function, i.e. $g^{2}$. This more stringent condition reads

$$
\frac{g^{4}}{16 \pi} \frac{\Lambda_{\mathrm{UV}}}{Q} \ll g^{2} \quad, \quad \text { i.e. } \Lambda_{\mathrm{UV}} \ll \frac{16 \pi Q}{g^{2}},
$$

where we have reintroduced the factors 2 and $\pi$ from eq. (42), because in practical situations they are numerically important. One can see that this inequality is easy to satisfy at weak coupling $g^{2} \ll 1$, and presumably only marginally satisfied at larger couplings $g \approx 1$.

\subsection{Ultraviolet contamination at asymptotic times}

The condition of eq. (52) ensures that the pathological NNLO contributions are much smaller than the NLO corrections (the latter are correctly given by the classical statistical approximation). Another important aspect of this discussion is whether, by ensuring that the inequality (52) is satisfied, one is guaranteed that the contamination by the pathological terms remains small at all times. It is easy to convince oneself that this is not the case. In Ref. [78], we argue that these pathological terms, if not removed, induce corrections that become comparable to the physical result after a time that varies as $Q t_{*} \sim 2048 \pi^{3} g^{-4}\left(Q / \Lambda_{\mathrm{UV}}\right)^{2}$. Effectively, these ultraviolet divergent terms act as spurious scatterings with a rate proportional to $g^{4} \Lambda_{\mathrm{UV}}^{2} / Q$.

Moreover, the state reached by the system when $t \rightarrow+\infty$ is controlled solely by conservation laws and by a few quantities that characterize the initial condition, in addition to the ultraviolet cutoff. For instance, if the only conserved quantities are energy and momentum, then the asymptotic state depends only on the total energy in the system. If in addition the particle number was conserved, then the asymptotic state would also depend on the number of particles in the system.

In particular, the value of the coupling constant does not play a role in determining which state is reached at asymptotic times; it only controls how quickly the system approaches the asymptotic state. This means that, even if $g^{2}$ is small so that the inequality (52) is satisfied, the CSA may evolve the system towards an asymptotic state that differs significantly from the true asymptotic state, regardless of how small $g^{2}$ is. Therefore, the strong dependence of the asymptotic state on the ultraviolet cutoff observed in the figure 10 of Ref. [5] is not specific to a "large" coupling $g^{2}=1$. Exactly the same cutoff dependence would be observed at smaller couplings, but the system would need to evolve for a longer time in order to reach it.

\subsection{Could it be fixed?}

An important issue is whether one could somehow alter the classical statistical approximation in order to remove the linear divergence that appears in the 1- 
loop 4-point function $\Gamma_{1122}$. As a support of these considerations, let us consider the NNLO correction to the function $G_{22}$. Eq. (48) displays the unique contribution in the classical statistical approximation. However, in the full theory there are two other possible arrangements of the internal 1/2 inside the 1122 subdiagram. This topology with the complete 1122 subdiagram reads :
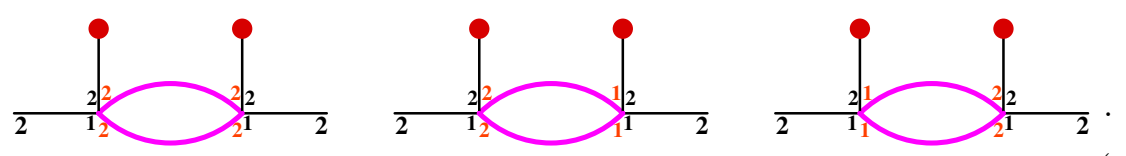

The CSA result contains only the first graph, and as a consequence it has a linear ultraviolet divergence, while the sum of the three graphs is finite. So the question is: could one reintroduce in the CSA the divergent part of the 2nd and 3rd graphs, in order to compensate the divergence of the first graph?

In order to better visualize what it would take to do this, let us modify the way the graphs are represented, so that they reflect the space-time evolution of the system and the modus operandi of practical implementations of the CSA. The modified representation for the first term in eq. (53) is shown in the figure 2. In this representation, the lines with arrows are retarded propagators (the

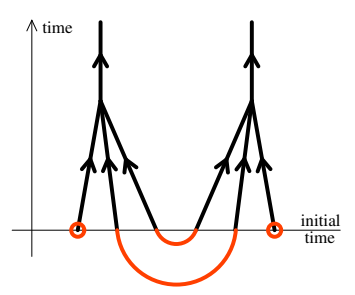

Figure 2: Space-time representation of eq. (48). The propagators with an arrow are retarded propagators. The orange circles represent the mean value of the initial field. The orange lines represent the link coming from the Gaussian fluctuations of the initial field.

time flows in the direction of the arrow). The solution of the classical equation of motion is a sum of trees made of retarded propagators, where the "leaves" of the tree are anchored to the initial surface. In the diagram shown in the figure 2, there are two such trees, both containing one instance of the quartic interaction term. In order to complete the calculation in the CSA, one performs a Gaussian average over the initial value of the classical field. Diagrammatically, this average amounts to attaching the leaves of the tree to 1-point objects representing the average value of the initial field, or to connecting them pairwise with the 2-point function that describes the variance of the initial Gaussian distribution.

It is crucial to note that the trees that appear in the solution of the classical equation of motion are "oriented" : three retarded propagators can merge at a point, from which a new retarded propagator starts. Let us call this a $3 \rightarrow 1$ vertex (when read in the direction of increasing time). These trees do not 
contain any $2 \rightarrow 2$ or $1 \rightarrow 3$ vertices. Their absence is intimately related to the absence of the 1122 and 1112 vertices in the Lagrangian in the classical statistical approximation.

In the figure 3, we now show the same representation for the 2nd and 3rd contributions of eq. (53). Firstly, we see that these graphs contain a $1 \rightarrow 3$ vertex (surrounded by a dotted circle in the figure), in agreement with the fact that they do not appear in the CSA. There is no way to generate the loop

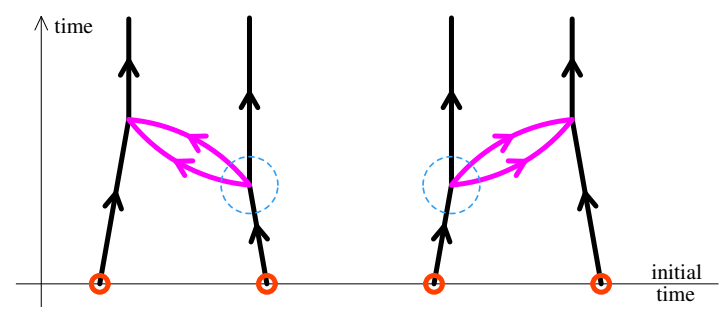

Figure 3: Space-time representation of the NNLO contributions to $G_{22}$ that are not included in the classical statistical approximation. The dotted circles outline the 1112 vertices, that are missing in the CSA.

contained in this graphs via the average over the initial conditions, because this loop corresponds to quantum fluctuations that happen later on in the time evolution. By Fourier transforming the divergent part of these diagrams in eq. (44), we can readily see that it is proportional to

$$
\frac{1}{|\boldsymbol{x}-\boldsymbol{y}|} \delta\left(\left(x^{0}-y^{0}\right)^{2}-(\boldsymbol{x}-\boldsymbol{y})^{2}\right)
$$

in coordinate space. Thus, the divergent part of these loops is non-local, with support on the light-cone, as illustrated in the figure 4.

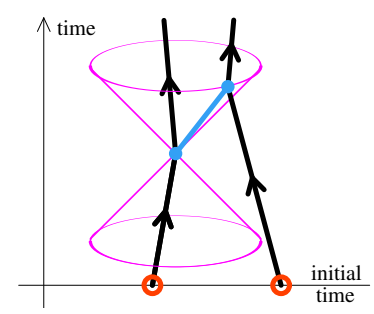

Figure 4: Space-time representation of the divergent part of the graphs of figure 3. As explained in the text, these divergent terms are non-local in space-time, with support on the light-cone.

There can also be arbitrarily many occurrences of these divergent subgraphs in the calculation of an observable in the classical statistical method, as illustrated in the figure 5 in the case of $G_{22}$. This implies that these divergences 


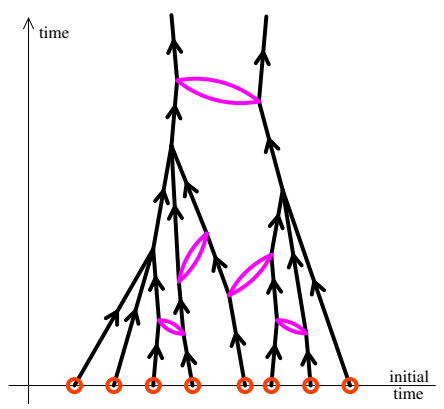

Figure 5: Contribution to $G_{22}$ with many $\Gamma_{1122}$ subgraphs.

cannot be removed by an overall subtraction, and that one must instead modify the Lagrangian. One could formally subtract them by adding to the action of the theory a non-local counterterm ${ }^{13}$ of the form

$$
\Delta \mathcal{S} \equiv-\frac{i}{2} \int \mathrm{d}^{4} x \mathrm{~d}^{4} y\left[\phi_{1}(x) \phi_{2}(x)\right] v(x, y)\left[\phi_{1}(y) \phi_{2}(y)\right],
$$

where

$$
v(x, y) \equiv \frac{g^{4}}{64 \pi^{3}} \frac{\Lambda_{\mathrm{UV}}}{|\boldsymbol{x}-\boldsymbol{y}|} \delta\left(\left(x^{0}-y^{0}\right)^{2}-(\boldsymbol{x}-\boldsymbol{y})^{2}\right)
$$

is tuned precisely to cancel the linear divergence in the $\Gamma_{1122}$ function. In order to deal with such a term, the simplest is to perform a Hubbard-Stratonovich transformation [90, 91], by introducing an auxiliary field $\zeta(x)$ via the following identity

$$
e^{i \Delta \mathcal{S}}=\int[\mathrm{D} \zeta] e^{\frac{1}{2} \int_{x, y} \zeta(x) v^{-1}(x, y) \zeta(y)} e^{i \int_{x} \zeta(x) \phi_{1}(x) \phi_{2}(x)} .
$$

The advantage of this transformation is that we have transformed a non-local four-field interaction term into a local interaction with a random Gaussian auxiliary field.

The rest of the derivation of the classical statistical method remains the same: the field $\phi_{1}$ appears as a Lagrange multiplier for a classical equation of motion for the field $\varphi$, but now we get an extra, stochastic, term in this equation :

$$
\left(\square+m^{2}\right) \varphi+\frac{g^{2}}{6} \varphi^{3}+i \xi \varphi=j
$$

Note that we have introduced $\zeta \equiv i \xi$ in order to have a positive definite variance for the new variable $\xi$. From the above derivation, this noise must be Gaussian

\footnotetext{
${ }^{13}$ Of course, there was no $\phi_{1}^{2} \phi_{2}^{2}$ term in the original bare action. On the other hand, we know that in the full theory, there should not be an intrinsic ultraviolet divergence in the 1122 function. One should view this counterterm as a way of reintroducing some of the terms that are beyond the classical statistical approximation, in order to restore the finiteness of the 1122 function.
} 
distributed, with a mean and variance given by the following formulas,

$$
\begin{aligned}
\langle\xi(x)\rangle & =0 \\
\langle\xi(x) \xi(y)\rangle & =\frac{g^{4}}{64 \pi^{3}} \frac{\Lambda_{\mathrm{UV}}}{|\boldsymbol{x}-\boldsymbol{y}|} \delta\left(\left(x^{0}-y^{0}\right)^{2}-(\boldsymbol{x}-\boldsymbol{y})^{2}\right) .
\end{aligned}
$$

By construction, the noise term in eq. (58), once averaged with eq. (59), will insert a non-local counterterm in every place where the $\Gamma_{1122}$ function can appear. For instance, when applied to the calculation of $G_{22}$, the contribution shown in the figure 5 will be accompanied by the term shown in the figure 6 .

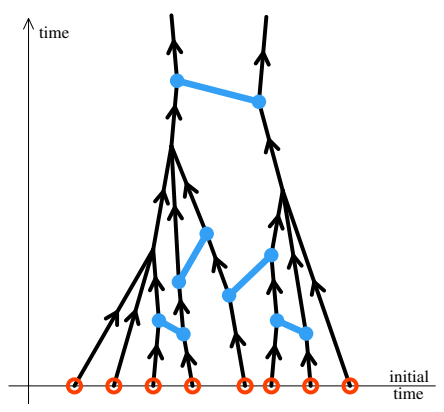

Figure 6: Effect of the noise term on the topology shown in the figure 5 .

Although the noise in eq. (59) has non-local space-time correlations, it is easy to generate it in momentum space, where it becomes diagonal. The main practical difficulty however comes from the non-locality in time ${ }^{14}$ : one would need to generate and store the whole spatio-temporal dependence for each configuration of the noise term prior to solving the modified classical equation of motion.

The noise term introduced in eq. (58) is purely imaginary, and it turns the classical field $\phi$ into a complex valued quantity. However, since $\xi$ is Gaussian distributed with a zero mean, any Hermitean observable constructed from $\phi$ via an average over $\xi$ will be real valued ${ }^{15}$. Eq. (58) is therefore a complex Langevin equation, and may be subject to the problems sometimes encountered with this kind of equations (lack of convergence, or convergence to the incorrect solution). At the moment, it is an open question whether eq. (58) really offers a practical way of removing the linear ultraviolet divergences from the classical statistical approximation.

\footnotetext{
${ }^{14}$ This non-locality appears to be a reminiscence of the memory effects that exist in the full quantum field theory, but are discarded in the classical statistical approximation. Note that the 2PI resummation scheme also has such terms.

${ }^{15}$ The average over $\xi$ will only retain terms that are even in $\xi$, and the factors $i$ will cancel.
} 


\section{Conclusions and outlook}

In this work, we have investigated the ultraviolet behavior of the classical statistical approximation. This has been done by using perturbation theory in the retarded-advanced basis, where this approximation has a very simple expression, and in calculating all the one-loop subdiagrams that can possibly be generated with these diagrammatic rules.

The main conclusion of this study is that the classical approximation leads to a 1-loop 4-point function that diverges linearly in the ultraviolet cutoff. More specifically, the problem lies in the function $\Gamma_{1122}$, where the 1,2 indices refer to the retarded-advanced basis. In the unapproximated theory, this function is ultraviolet finite, but it violates Weinberg's theorem in the classical statistical approximation, because it has an ultraviolet divergence with a coefficient which is non-polynomial in the external momenta. Moreover, it is non-renormalizable because it corresponds to an operator that does not even appear in the Lagrangian one started from.

The mere existence of these divergent terms implies that the classical statistical approximation is not renormalizable, no matter what the value of the coupling constant is.

We have estimated that the contamination of the results by these nonrenormalizable terms is of relative order $g^{4} \Lambda_{\mathrm{UV}} / Q$, where $Q$ is the typical physical momentum scale of the problem under consideration. Based on this, the general rule is that the coupling should not be too large, and the cutoff should remain close enough to the physical scales.

In this paper, we have also proposed that this one-loop spurious (because it does not exist in the full theory) divergence may be subtracted by adding a multiplicative Gaussian noise term to the classical equation of motion. This noise term can be tuned in order to reintroduce some of the terms of the full theory that had been lost when doing the classical approximation. In order to subtract the appropriate quantity, this noise must be purely imaginary, with a 2-point correlation given by the Fourier transform of the divergent term. Unfortunately, this correlation is non-local in time, which makes the implementation of this correction quite complicated. Whether this can be done in practice remains an open question at this point.

Moreover, the ultraviolet contamination due to these non-renormalizable terms is cumulative over time, and will eventually dominate the dynamics of the system no matter how small $g^{4} \Lambda_{\mathrm{UV}} / Q$ is. An extensive discussion of this asymptotic ultraviolet sensitivity, and of the time evolution that leads to the asymptotic state, will be provided in a forthcoming work [78].

\section{Acknowledgements}

We would like to thank L. McLerran for useful discussions about this work and closely related issues. This work is supported by the Agence Nationale de la Recherche project 11-BS04-015-01. 


\section{A $\Gamma_{1222}$ and $\Gamma_{1112}$ at one loop}

At one loop, the 4-point functions $\Gamma_{1222}$ and $\Gamma_{1112}$ are given by the following sets of graphs :

$$
-i \Gamma_{1222}^{1 \text { loop }\left(p_{1} \cdots p_{4}\right)=}{ }_{-i \Gamma_{2111}^{1 \text { loop }}\left(p_{1} \cdots p_{4}\right)}={ }_{\mathrm{p}_{2}}=-i\left[I\left(p_{3}+p_{4}\right)+I\left(p_{2}+p_{3}\right)+I\left(p_{2}+p_{4}\right)\right],
$$

Note that all the 1-loop contributions to $\Gamma_{1112}$ are zero if we exclude the 1112 vertex, as done in the classical statistical approximation. The fact that $\Gamma_{1222}$ and $\Gamma_{2111}$ differ only by a factor $1 / 4$ is a consequence of their common origin in the Schwinger-Keldysh formalism, where the ++++ and ---- vertex functions are mutual complex conjugates.

They are all expressible in terms of a single loop integral,

$$
I(P) \equiv-i g^{4} \int \frac{\mathrm{d}^{4} K}{(2 \pi)^{4}} G_{22}(K) G_{12}(P+K) .
$$

Since $G_{22}(K)$ is a delta function $\delta\left(K^{2}\right)$, we use it in order to perform the integration over the energy $k^{0}$. Then, the integration over $\cos (\theta)$, where $\theta$ is the angle between the 3 -vectors $\boldsymbol{p}$ and $\boldsymbol{k}$, is elementary but requires that one studies carefully whether $P^{2}+2 P \cdot K$ can vanish in the integration range. If this quantity can vanish, the integral will also have an imaginary part. This leads to the following expression for $I(P)$ :

$$
\begin{array}{r}
I(P)=\frac{g^{4}}{32 \pi^{2}}\left\{\frac{1}{p} \int_{0}^{\Lambda_{\mathrm{UV}}} \mathrm{d} k \sum_{\epsilon= \pm 1} \log \left|\frac{p+\epsilon p^{0}+P^{2} / 2 k}{p-\epsilon p^{0}-P^{2} / 2 k}\right|\right. \\
\left.\quad+i \pi\left(\theta\left(P^{2}\right) \operatorname{sign}\left(p^{0}\right)-\theta\left(-P^{2}\right) \frac{p^{0}}{p}\right)\right\} \\
=\frac{g^{4}}{32 \pi^{2}}\left\{\ln \left|\frac{P^{2}}{4 \Lambda_{\mathrm{UV}}^{2}}\right|+\frac{p^{0}}{p} \log \left|\frac{p^{0}+p}{p^{0}-p}\right|-2\right. \\
\left.+i \pi\left(\theta\left(P^{2}\right) \operatorname{sign}\left(p^{0}\right)-\theta\left(-P^{2}\right) \frac{p^{0}}{p}\right)\right\} .
\end{array}
$$


Therefore, the real part is logarithmically ultraviolet divergent, while the imaginary part is finite.

\section{B $\Gamma_{1122}$ at one loop}

In this appendix, we perform the calculation of some of the graphs contributing to $\Gamma_{1122}^{1}$ loop . The list of all the relevant graphs is given in eq. (40). The unique S-channel graph is zero, because it has a closed loop of retarded propagators. Since the T- and U-channel graphs are identical, up to the permutation $p_{3} \leftrightarrow p_{4}$, we will calculate only the T-channel graphs here.

\section{B.1 Classical statistical approximation at one-loop}

Let us consider first the graph the contributes in the classical statistical approximation :

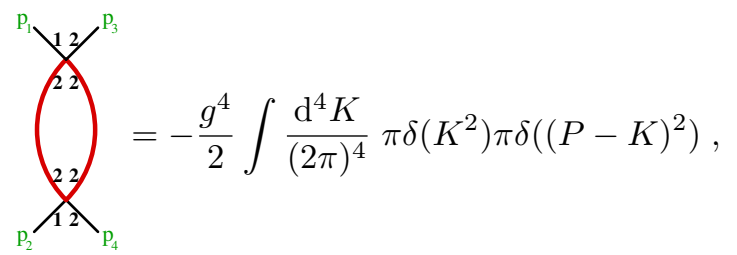

where we denote $P \equiv p_{1}+p_{3}$. We first use the $\delta\left(K^{2}\right)$ in order to perform the integral over $k^{0}$, which gives

$$
\int_{22}^{\mathrm{P}_{2}}=-\frac{g^{4}}{32 \pi} \int_{0}^{\Lambda_{\mathrm{Uv}}} k \mathrm{~d} k \int_{-1}^{+1} \mathrm{~d}(\cos \theta) \sum_{\epsilon= \pm} \delta\left(P^{2}-2 \epsilon k p^{0}+2 p k \cos \theta\right) \text {, }
$$

Anticipating the fact that the integral over $k=|\boldsymbol{k}|$ is ultraviolet divergent, we have introduced an upper cutoff on this integral. The second step is to use the remaining delta function in order to integrate over $\cos \theta$. This requires some careful analysis, in order to determine whether there is a valid solution, i.e. one for which $-1 \leq \cos \theta \leq+1$. This depends on the sign $\epsilon$, on the sign of $P^{2}$ and on the value of $k$. The results are summarized here :

- If $P^{2}>0$, there is a valid solution if and only if :

$$
\epsilon p^{0}>0 \text { and } \frac{\left|p^{0}\right|-p}{2} \leq k \leq \frac{\left|p^{0}\right|+p}{2},
$$

- If $P^{2}<0$ :

- There is no solution if $k<\frac{p-\left|p^{0}\right|}{2}$ 
- If $\epsilon p^{0}<0$, there is a solution if $\frac{p-\left|p^{0}\right|}{2} \leq k$
- If $\epsilon p^{0}>0$, there is a solution if $\frac{p+\left|p^{0}\right|}{2} \leq k$

Using this, the integration over $\cos \theta$ leads to a piecewise constant integrand for the remaining integral. We eventually obtain

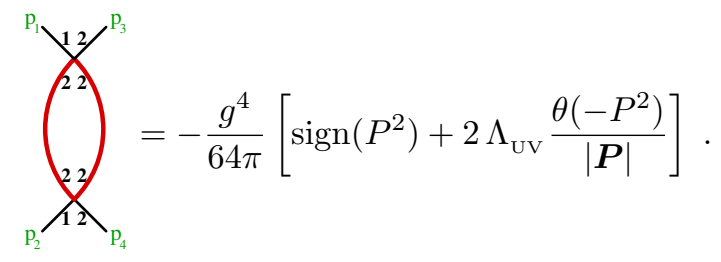

\section{B.2 One-loop graphs beyond the CSA}

Let us now focus on the 2nd and 3rd T-channel graphs,

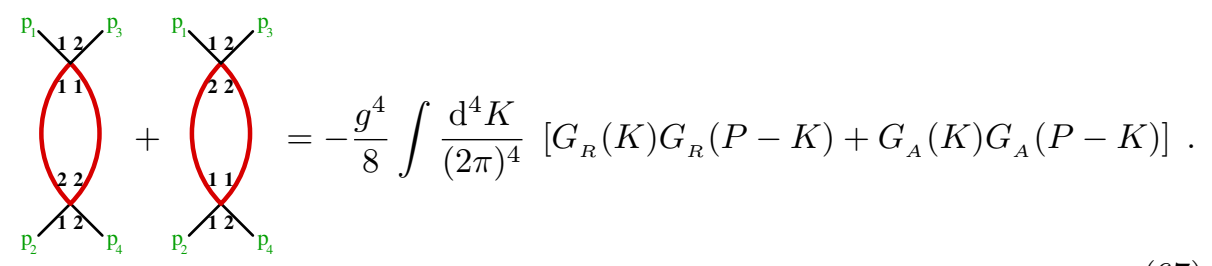

Changing $K^{\mu} \rightarrow-K^{\mu}$ in the second term, we see that this term is the same as the first one with the change $P^{\mu} \rightarrow-P^{\mu}$. Therefore, we need only to calculate the first term, multiply by two its $P$-even part and discard its $P$-odd part. The first step is to perform the $k^{0}$ integral in the complex plane. By closing the integration contour in the lowest half-plane, we pick the two poles of $G_{R}(K)$, and we get

$$
\left(\int_{22}^{\mathrm{P}_{2}}=-\frac{g^{4}}{64 \pi^{2}} \int_{0}^{\Lambda_{\mathrm{UV}}} k \mathrm{~d} k \int_{-1}^{+1} \mathrm{~d}(\cos \theta) \sum_{\epsilon= \pm}^{\mathrm{P}_{3}} \epsilon\left[G_{R}(P-K)\right]_{k^{0}=\epsilon k} .\right.
$$

At this point, one can decompose the retarded propagator into a principal value term and a delta function. One can check that the principal value gives only terms that are $P$-odd, that we can thus drop. Keeping only the delta function leads to

$$
\int_{22}^{\mathrm{m}_{2}}=-\frac{g^{4}}{64 \pi} \int_{0}^{\Lambda_{\mathrm{UV}}} k \mathrm{~d} k \int_{-1}^{+1} \mathrm{~d}(\cos \theta) \sum_{\epsilon= \pm} \operatorname{sign}\left(\epsilon p^{0}-k\right) \delta\left(P^{2}-2 \epsilon k p^{0}+2 p k \cos \theta\right) \text {. }
$$


We proceed by using the delta function to perform the integral over $\cos \theta$. The conditions for having a valid solution are the same as before. Finally, we obtain

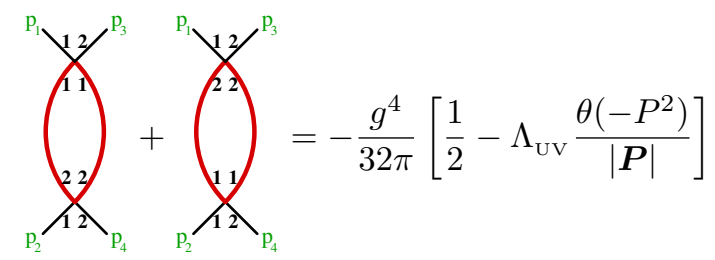

\section{B.3 CSA result with symmetric regularization}

The ultraviolet regularization introduced in eq. (65) is not entirely satisfactory, because it breaks the symmetry between the two internal lines of the graph, by placing a cutoff $\Lambda_{\mathrm{UV}}$ on the 3-momentum $|\boldsymbol{k}|$, while the 3 -momentum $|\boldsymbol{p}-\boldsymbol{k}|$ in the other internal line is not constrained.

It is perfectly fine to do so. However, some important identities obeyed by 2-loop self-energies, in which the 1-loop $\Gamma_{1122}$ function appears as a subgraph, rely on the symmetry between the internal lines of the graph. It is therefore important that the regularization scheme employed in intermediate steps of the calculation does not break this symmetry, and in particular that the subgraph itself respects this symmetry. This issue will be discussed at length in a forthcoming work, Ref. [78], but for later reference we present here the formulas for the function $\Gamma_{1122}$ at 1-loop with a symmetric regularization. This just amounts to replacing eq. (65) by

$$
\int_{22}^{\mathrm{N}_{2}}=-\frac{g^{4}}{32 \pi} \int k \mathrm{~d} k \int_{-1}^{+1} \mathrm{~d}(\cos \theta) \sum_{\epsilon= \pm} \delta\left(P^{2}-2 \epsilon k p^{0}+2 p k \cos \theta\right)
$$

with $|\boldsymbol{p}-\boldsymbol{k}|=\left(p^{2}+k^{2}-2 p k \cos \theta\right)^{1 / 2}$. The new constraint on $|\boldsymbol{p}-\boldsymbol{k}|$ slightly complicates the discussion of the various cases, and in the end we obtain:

$$
-\frac{g^{4}}{32 \pi p} \times\left\{\begin{array}{lr}
{\left[\Lambda_{\mathrm{UV}}-\frac{p+\left|p_{0}\right|}{2}\right] \theta\left(\Lambda_{\mathrm{UV}}-\frac{p+\left|p_{0}\right|}{2}\right)} & {\left[P^{2}<0\right]} \\
\frac{p}{2} & {\left[P^{2}>0, \frac{p+\left|p_{0}\right|}{2} \leq \Lambda_{\mathrm{UV}}\right]} \\
\Lambda_{\mathrm{UV}}-\frac{\left|p_{0}\right|}{2} & {\left[P^{2}>0, \frac{\left|p_{0}\right|}{2} \leq \Lambda_{\mathrm{UV}} \leq \frac{p+\left|p_{0}\right|}{2}\right]} \\
0 & {\left[P^{2}>0, \Lambda_{\mathrm{UV}} \leq \frac{\left|p_{0}\right|}{2}\right]}
\end{array}\right.
$$

instead of eq. (66). Note that the ultraviolet divergence itself (i.e. the terms that diverge when $\Lambda_{\mathrm{UV}} \rightarrow+\infty$ at fixed $P^{\mu}$ ) is not affected by this modification of the regularization procedure. 


\section{References}

[1] J. Berges, J.P. Blaizot, F. Gelis, J. Phys. G 39, 085115 (2012).

[2] J. Berges, K. Boguslavski, S. Schlichting, Phys. Rev. D 85, 076005 (2012).

[3] J. Berges, K. Boguslavski, S. Schlichting, R. Venugopalan, arXiv:1303.5650.

[4] J. Berges, K. Boguslavski, S. Schlichting, R. Venugopalan, arXiv:1311.3005.

[5] J. Berges, K. Boguslavski, S. Schlichting, R. Venugopalan, arXiv:1312.5216.

[6] J. Berges, D. Gelfand, S. Scheffler, D. Sexty, Phys. Lett. B 677, 210 (2009).

[7] J. Berges, D. Sexty, Phys. Rev. Lett. 108, 161601 (2012).

[8] J. Berges, S. Schlichting, D. Sexty, Phys. Rev. D 86, 074006 (2012).

[9] K. Fukushima, F. Gelis, Nucl. Phys. A 874, 108 (2012).

[10] K. Fukushima, arXiv:1307.1046.

[11] T. Kunihiro, B. Muller, A. Ohnishi, A. Schafer, T.T. Takahashi, A Yamamoto, Phys. Rev. D 82, 114015 (2010).

[12] H. Iida, T. Kunihiro, B. Mueller, A. Ohnishi, A. Schaefer, T.T. Takahashi, Phys. Rev. D 88, 094006 (2013).

[13] K. Dusling, T. Epelbaum, F. Gelis, R. Venugopalan, Nucl. Phys. A 850, 69 (2011).

[14] T. Epelbaum, F. Gelis, Nucl. Phys. A 872, 210 (2011).

[15] K. Dusling, T. Epelbaum, F. Gelis, R. Venugopalan, Phys. Rev. D 86, 085040 (2012).

[16] T. Epelbaum, F. Gelis, Phys. Rev. D 88, 085015 (2013).

[17] T. Epelbaum, F. Gelis, Phys. Rev. Lett. 111, 232301 (2013).

[18] A. Kurkela, G.D. Moore, JHEP 1112, 044 (2011).

[19] A. Kurkela, G.D. Moore, JHEP 1111, 120 (2011).

[20] A. Kurkela, G.D. Moore, Phys. Rev. D 86, 056008 (2012).

[21] M.C. Abraao York, A. Kurkela, E. Lu, G.D. Moore, arXiv:1401.3751.

[22] M. Attems, A. Rebhan, M. Strickland, Phys. Rev. D 87, 025010 (2013).

[23] J.P. Blaizot, F. Gelis, J. Liao, L. McLerran, R. Venugopalan, Nucl. Phys. A 873, 68 (2012).

[24] J.P. Blaizot, J. Liao, L.D. McLerran, arXiv:1305.2119. 
[25] M.P. Heller, R.A. Janik, P. Witaszczyk, Phys. Rev. Lett. 108, 201602 (2012).

[26] J. Casalderrey-Solana, M.P. Heller, D. Mateos, W. van der Schee, Phys. Rev. Lett. 111, 181601 (2013).

[27] B. Wu, JHEP 1304, 044 (2013).

[28] U. Reinosa, J. Serreau, Annals Phys. 325, 969 (2010).

[29] F. Gautier, J. Serreau, Phys. Rev. D 86, 125002 (2012).

[30] A. Giraud, J. Serreau, Phys. Rev. Lett. 104, 230405 (2010).

[31] S. Floerchinger, C. Wetterich, arXiv:1311.5389.

[32] T. Gasenzer, J.M. Pawlowski, Phys. Lett. B 670, 135 (2008).

[33] T. Gasenzer, S. Kessler, J.M. Pawlowski, Eur. Phys. J. C 70, 423 (2010).

[34] P. Arnold, G.D. Moore, L.G. Yaffe, JHEP 0301, 030 (2003).

[35] J.M. Luttinger, J.C. Ward, Phys. Rev. 118, 1417 (1960).

[36] G. Baym, L.P. Kadanoff, Phys. Rev. 124, 287 (1961).

[37] C. de Dominicis, P.C. Martin, J. Math. Phys. 5, 14 (1964).

[38] C. de Dominicis, P.C. Martin, J. Math. Phys. 5, 31 (1964).

[39] J.M. Cornwall, R. Jackiw, E. Tomboulis, Phys. Rev. D 10, 2428 (1974).

[40] J. Berges, AIP Conf. Proc. 739, 3 (2005).

[41] J. Berges, S. Borsányi, U. Reinosa, J. Serreau, Annals Phys. 320, 344 (2005).

[42] U. Reinosa, J. Serreau, JHEP 0607, 028 (2006).

[43] A. Branschadel, T. Gasenzer, J. Phys. B 41, 135302 (2008).

[44] G. Aarts, N. Laurie, A. Tranberg, Phys. Rev. D 78125028 (2008).

[45] Y. Hatta, A. Nishiyama, Nucl. Phys. A 873, 47 (2012).

[46] Y. Hatta, A. Nishiyama, Phys.Rev. D 86, 076002 (2012).

[47] E. Iancu, A. Leonidov, L.D. McLerran, Lectures given at Cargese Summer School on QCD Perspectives on Hot and Dense Matter, Cargese, France, 6-18 Aug 2001, hep-ph/0202270.

[48] E. Iancu, R. Venugopalan, Quark Gluon Plasma 3, Eds. R.C. Hwa and X.N. Wang, World Scientific, hep-ph/0303204. 
[49] T. Lappi, L.D. McLerran, Nucl. Phys. A 772, 200 (2006).

[50] F. Gelis, E. Iancu, J. Jalilian-Marian, R. Venugopalan, Ann. Rev. Part. Nucl. Sci. 60, 463 (2010).

[51] F. Gelis, Int. J. Mod. Phys. A 28, 1330001 (2013).

[52] D. Polarski, A.A. Starobinsky, Class. Quant. Grav. 13, 377 (1996).

[53] D.T. Son, hep-ph/9601377.

[54] S.Yu. Khlebnikov, I.I. Tkachev, Phys. Rev. Lett. 77, 219 (1996).

[55] F. Gelis, T. Lappi, R. Venugopalan, Int. J. Mod. Phys. E 16, 2595 (2007).

[56] K. Fukushima, F. Gelis, L. McLerran, Nucl. Phys. A 786, 107 (2007).

[57] S. Jeon, Annals Phys. 340, 119 (2014).

[58] D. Bodeker, L.D. McLerran, A. Smilga, Phys. Rev. D 52, 4675 (1995).

[59] P. Arnold, D.T. Son, L.G. Yaffe, Phys. Rev. D 55, 6264 (1997).

[60] G. Aarts, J. Smit, Phys. Lett. B 393, 395 (1997).

[61] G. Aarts, J. Smit, Nucl. Phys. B 511, 451 (1998).

[62] G. Aarts, B.J. Nauta, C.G. van Weert, Phys. Rev. D 61, 105002 (2000).

[63] D.Y. Grigoriev, V.A. Rubakov, Nucl. Phys. B 299, 67 (1988).

[64] D.Y. Grigoriev, V.A. Rubakov, M.E. Shaposhnikov, Nucl. Phys. B 326, 737 (1989).

[65] D.Y. Grigoriev, V.A. Rubakov, M.E. Shaposhnikov, Phys. Lett. B 216, 172 (1989).

[66] J. Ambjorn, M. Laursen, M.E. Shaposhnikov, Phys. Lett. B 197, 49 (1987).

[67] J. Ambjorn, M. Laursen, M.E. Shaposhnikov, Nucl. Phys. B 316, 483 (1989).

[68] J. Ambjorn, T. Askgaard, H. Porter, M.E. Shaposhnikov, Phys. Lett. B 244, 479 (1990).

[69] J. Ambjorn, T. Askgaard, H. Porter, M.E. Shaposhnikov, Nucl. Phys. B 353, 346 (1991).

[70] J. Ambjorn, K. Farakos, Phys. Lett. B 294, 248 (1992).

[71] J. Schwinger, J. Math. Phys. 2, 407 (1961).

[72] L.V. Keldysh, Sov. Phys. JETP 20, 1018 (1964). 
[73] P. Aurenche, T. Becherrawy, Nucl. Phys. B 379, 259 (1992).

[74] M.A. van Eijck, R. Kobes, Ch.G. van Weert, Phys. Rev. D 50, 4097 (1994).

[75] F. Gelis, Nucl. Phys. B 508, 483 (1997).

[76] F. Gelis, T. Lappi, R. Venugopalan, Phys. Rev. D 78, 054019 (2008).

[77] F. Gelis, T. Lappi, R. Venugopalan, Phys. Rev. D 78, 054020 (2008).

[78] T. Epelbaum, F. Gelis, N. Tanji, B. Wu, In preparation.

[79] R. Kubo, J. Phys. Soc. Japan 12, 570 (1957).

[80] P.C. Martin, J. Schwinger, Phys. Rev. 115, 1342 (1959).

[81] F.J. Dyson, Phys. Rev. 75, 1736 (1949).

[82] S. Weinberg, Phys. Rev. 111, 838 (1960).

[83] Y. Hahn, W. Zimmermann, Comm. Math. Phys. 10, 330 (1968).

[84] W. Zimmermann, Comm. Math. Phys. 11, 1 (1968).

[85] W.E. Caswell, A.D. Kennedy, Phys. Rev. D 25, 392 (1982).

[86] W.E. Caswell, A.D. Kennedy, Phys. Rev. D 28, 3073 (1983).

[87] J.C. Collins, Renormalization, Cambridge University Press (1984).

[88] R.E. Cutkosky, J. Math. Phys. 1, 429 (1960).

[89] G. t'Hooft, M.J.G. Veltman, CERN report 73-9.

[90] J. Hubbard, Phys. Rev. Lett. 3, 77 (1959).

[91] R.L. Stratonovich, Sov. Phys. Dok. 2416 (1957). 